Relations industrielles

Industrial Relations

\title{
Gabriel TAHAR : La réduction de la durée du travail. Paris, Collection Repères, Éditions la découverte, 1985, 127 pp., ISBN 2-7071-1552-5
}

\section{Lise Chrétien}

Volume 42, numéro 3, 1987

URI : https://id.erudit.org/iderudit/050343ar

DOI : https://doi.org/10.7202/050343ar

Aller au sommaire du numéro

Éditeur(s)

Département des relations industrielles de l'Université Laval

ISSN

0034-379X (imprimé)

1703-8138 (numérique)

Découvrir la revue

Citer ce compte rendu

Chrétien, L. (1987). Compte rendu de [Gabriel TAHAR : La réduction de la durée du travail. Paris, Collection Repères, Éditions la découverte, 1985, 127 pp., ISBN 2-7071-1552-5]. Relations industrielles / Industrial Relations, 42(3), 646-648.

https://doi.org/10.7202/050343ar

Tous droits réservés @ Département des relations industrielles de l'Université Laval, 1987
Ce document est protégé par la loi sur le droit d'auteur. L’utilisation des services d'Érudit (y compris la reproduction) est assujettie à sa politique d'utilisation que vous pouvez consulter en ligne.

https://apropos.erudit.org/fr/usagers/politique-dutilisation/ 
sur le plan théorique que méthodologique, retenir l'attention de tous ceux et celles qui se préoccupent de l'analyse des politiques sociales et des pratiques de l'État. Le défi relevé par l'auteure est à la mesure de la question. $\bar{A}$ un moment où $40 \%$ des dépenses sociales des pays industrialisés sont consacrées à la retraite (p. 316), Anne-Marie Guillemard a fait la démonstration magistrale que, moins que jamais, la gérontologie sociale ne doit être réduite à l'examen des conduites individuelles des personnes âgées. Son ouvrage démontre la nécessité d'amorcer et de poursuivre des travaux sur les politiques de la vieillesse, tant publiques que privées, dans une grande variété des contextes économiques et politiques.

Hélène DAVID

Institut de recherche appliquée

sur le travail

BINSTOCK, Robert H., «Interest Group Liberalism and the Politics of Aging», Gerontologist, Vol. 12, 1972.

CRIBIER, Françoise, «Itinéraires professionnels et usure au travail: une génération de salariés parisiens», dans Le mouvement social, no 124, 1983, pp. 11-44.

DOWD, James J., Stratification Among the Aged, Monterey, Brooks/Cole, 1980.

ESTES, Carroll, The Aging Entreprise, San Francisco, Jossey - Bass Publishers, 1979.

FROSSARD, Michel, «Crise et cessation anticipées d'activité: une comparaison internationale», Travail et emploi, no 16, avril-juin 1983, pp. 27-36.

GAULLIER, Xavier, «Âge et emploi: les salariés âgés et la fin de carrière», Revue française des affaires sociales, no 1, 1986. GRAEBNER, William, A History of Retirement: the Meaning and Function of an American Institution, New Haven, Yale University Press, 1980.

GUILLEMARD, Anne-Marie, (sous la direction de) Old Age and the Welfare State, Beverley Hills, Sage, 1983.

McDONALD, P. Lynn et Richard A. WANNER, «Retirement in a Dual Economy: The Canadian Case», pp. 245 et 261 dans Aging in Canada, sous la direction de Victor W. Marshall, Toronto, Fitzhenry and Whiteside 1987 ( $2^{\mathrm{e}}$ édition).

MYLES, John F., "The Aged, the State and the Structure of Inequality», pp. 317 a 342 dans Structured Inequality in Canada, sous la direction de John Harp et John Hofley, Scarborough, Prentice-Hall, 1980.

PHILlIPSON, Chris, Capitalism and the Construction of Old Age, Londres, the MacMillan Press Ltd., 1982.

PRATT, Henry J., The Gray Lobby, Chicago, Chicago University Press, 1976.

WALKER, Alan, «Towards a Political Economy of Old Age», Aging and Society, vol. 1, no 1, 1981, pp. $73-94$.

La réduction de la durée du travail, par Gabriel Tahar, Paris, Collection Repères, Éditions La Découverte, 1985, 127 pp., ISBN 2-7071-1552-5

«Les professions de foi et analyses plus ou moins simplistes sur l'évolution du temps de travail n'ont pas manqué ces dernières années.» C'est ainsi que $\mathrm{G}$. Tahar débute son livre sur la réduction de la durée du travail (RDT). Il se devait donc d'éviter à tout prix de tomber luimême dans ce piège. Après lecture, on peut affirmer qu'il a réussi.

L'analyse de la RDT proposée par Tahar est centrée sur la société duale, ce qui revient à se demander si on doit accepter que dans nos sociétés vivent côte-à-côte des salariés à niveau de vie élevé et des chômeurs laissés pour compte? Examiner la RDT en orientant l'analyse sur l'emploi et le revenu permet de la resituer par rapport aux différents projets de société. Selon l'auteur, les deux principales thèses qui s'affrontent sont le mouvement écologiste pour lequel le progrès social ne passe pas par n'importe lequel accroissement de la production et le courant productiviste selon lequel il faut accélérer le développement de la société marchande pour satisfaire nos larges besoins. 
À partir de la variable «emploi», l'auteur divise son analyse de la RDT en quatre parties. Dans la première partie, il explique comment, dans le passé, la RDT a été une source de progrès social important et comment elle a permis, à une époque plus récente, de ne pas perdre trop d'emplois. Dans la deuxième partie, on s'interroge sur l'efficacité de la RDT à créer des emplois et sur les conditions économiques qui favorisent cette réussite. Dans la troisième partie, G. Tahar définit la durée du travail aujourd'hui et explique les luttes sociales dont elle fait l'objet. Finalement, la quatrième partie a pour but de faire saisir l'importance de la RDT pour l'avenir.

Ainsi, dans la première partie, l'auteur présente une rétrospective de la durée du travail en France.

Deux principales conclusions se dégagent de l'analyse de l'auteur sur cette première partie de son volume. Tout d'abord, les interventions de l'Etat pour activer la RDT sont en régression. Elles ont eu le mérite de tracer la voie mais les décisions de RDT sont maintenant laissées aux partenaires sociaux en fonction de leurs besoins et de leurs possibilités. Une deuxième conclusion, et la plus importante, concerne le peu d'efficacité de la RDT en matière d'emploi. Les RDT que l'on a connues jusqu'à maintenant n'ont pas permis de résoudre le problème du chômage. Au mieux, elles ont évité des pertes d'emplois, ce qui n'est pas à négliger mais plutôt insuffisant.

Cette première partie présente surtout l'intérêt de bien décrire l'évolution historique de la RDT et d'en dégager les effets. L'auteur le fait de façon très objective en précisant autant les échecs que les succès. Il s'agit donc d'une source d'informations importante pour les lecteurs qui s'intéressent à l'évolution de la RDT en France. De plus, le cheminement analytique peut servir de modèle à ceux qui souhaitent le faire pour un autre pays. Les tableaux et schémas sont bien présentés et permettent de tirer rapidement l'information souhaitée.

Suite à ce bilan, l'auteur nous présente, en deuxième partie, une analyse microéconomique de la dynamique de créations d'emplois. Il tente de nous faire saisir les mécanismes qui associent durée du travail et emploi ainsi que les circonstances particulières qui ont limité l'impact de la RDT. On y analyse d'abord ce qu'est une heure de travail pour regarder ensuite les contraintes économiques et les préférences individuelles.

Contrairement à beaucoup de recherches sur la RDT qui ne consistent qu'à présenter et interpréter les résultats d'enquêtes, l'analyse de G. Tahar s'appuie sur de solides fondements théoriques. Dans cette deuxième partie, l'auteur expose brièvement et avec clarté son cadre d'analyse théorique et l'appuie des résultats de plusieurs recherches. La théorie est bien vulgarisée de façon à permettre au lecteur de suivre le raisonnement même s'il a peu de connaissances en économique. De plus, l'analyse microéconomique dynamique saura certainement intéresser tous ceux qui veulent mieux comprendre les choix qu'implique une RDT pour les entreprises et les individus. Cette partie constitue vraiment le point fort du volume.

Dans la troisième partie, l'auteur discute de la durée du travail aujourd'hui. ll aborde dans une première section les caractéristiques actuelles des temps travaillés et dans une deuxième section le débat social entourant la RDT à l'étranger et en France. G. Tahar met l'accet sur quatre caractéristiques principales dont il justifie ainsi le choix: «il faut s'intéresser aux conditions de travail qui précisent l'intensité et le contenu de l'heure de travail, au travail en équipes dont l'impact est déterminant dans la vie quotidienne, au temps partiel dont l'importance remet en cause la rigidité du temps complet. Quelques éléments de comparaison avec l'étranger montrent qu'aucun pays n'échappe à la RDT».

L'observation du débat social à l'étranger relativement à la RDT vise surtout à faire saisir au lecteur l'importance de l'environnement économique et social dans les attitudes des parte- 
naires sociaux et des pouvoirs publics vis-à-vis de la RDT. L'auteur veut montrer que l'expérience française n'est pas indépendante de ce qui se passe dans les autres pays. Pour ce faire, il analyse d'abord deux positions extrêmes,... «On peut opposer le désengagement de l'État canadien et le «volontarisme» de l'État belge qui impose des règles de négociation très strictes aux entreprises.» Il propose ensuite une vue d'ensemble des luttes sociales en Europe concernant la RDT. Il termine cette section en décrivant la position des divers syndicats français dans leurs revendications en matière de RDT. Il insiste sur le fait qu'alors que la CGT, FO et CFTC privilégient la défense du pouvoir d'achat, la CFDT donne la priorité à l'emploi.

Cette partie est le point faible du volume et ce, particulièrement à cause de l'inconsistance des données. Lorsqu'il traite des caractéristiques actuelles des temps travaillés, l'auteur aborde 4 aspects: les conditions de travail, le travail en équipes, le temps complet et le temps partiel et les comparaisons internationales relativement à la durée du travail. Les trois premiers éléments sont abordés en fonction de leur évolution en France alors que les comparaisons internationales se font entre le Royaume-Uni, la RFA, les Etats-Unis, le Japon, la Belgique, et la France bien sâr. Parfois, on ajoute des données pour le Canada et la Suisse mais ce, de façon exceptionnelle. De plus, dans certains tableaux, on compare les données de 1973 avec celles de 1981 alors que dans d'autres cas on compare l'année 1973 à 1982 . L'absence quasi-totale de statistiques sur la durée du travail au Canada surprend d'autant plus que l'auteur accorde au Canada une place importante dans la section suivante qui traite du débat social à l'étranger. Toutefois, son argumentation s'appuie alors sur très peu de statistiques et lorsqu'il y en a, l'auteur réfère à des enquêtes récentes du gouvernement du Québec sans préciser ses sources. Il existe pourtant beaucoup d'études et de données sur la durée du travail au Canada dont aurait pu s'inspirer l'auteur. Cette dernière section paraît aussi très mal structurée. Une simple lecture de titres en donne une idée: 1 . Le cas du Canada, 2. L'exemple de la Belgique, 3. Les luttes sociales en Europe, 4. Les positions des syndicats français.

Dans la quatrième et dernière partie, G. Tahar tente de situer la durée du travail actuelle et le débat social que suscite sa réduction par rapport aux perspectives macroéconomiques. La durée du travail étant à la fois une variable de production (au niveau de l'entreprise) et d'ajustement (au niveau social), il devient utile d'apprécier le bien-fondé d'une politique économique s'appuyant sur la RDT. Trois options sont possibles: on s'engage vers le partage du travail, on laisse se multiplier les entrées et les sorties de la population active, ou encore un mélange entre les deux éventualités selon qu'on assistera à une plus ou moins grande solidarité sociale.

L'auteur termine son étude par une brève conclusion dans laquelle il affirme que la réussite tient à une prise de conscience de l'État, des employeurs, des syndicats ainsi que de tous les citoyens de leurs responsabilités socio-économiques. Dans le contexte économique actuel, «ne pas privilégier l'emploi revient à rester dans le schéma traditionnel de la priorité au salaire, à freiner la RDT et à subir la société duale». Suit une courte bibliographie (24 titres) dont la majorité des références sont françaises.

La dernière partie de ce volume a surtout le mérite de résumer l'état de la réflexion sur le sujet depuis le début des années 1980.

Dans l'ensemble, sans qu'il ne fasse vraiment preuve d'originalité, ce volume présente l'intérêt de résumer succintement l'état actuel des travaux d'analyse économique de la RDT. C'est donc un outil bien fait et utile que voudront lire tous ceux qui se préoccupent des politiques de durée du travail comme mesures de création d'emplois.

Lise CHRÉTIEN 